\title{
Measurement of thermal contact conductance between round-shaped superconducting wires and rectangular slot in copper block for application to cryogenic transfer tube
}

Taekyung Ki ${ }^{\mathrm{a}}$, Makoto Yoshida ${ }^{\mathrm{b}}$, Ye Yang ${ }^{\mathrm{a}, \mathrm{c}}$, Toru Ogitsu ${ }^{\mathrm{a}}$, Nobuhiro Kimura ${ }^{\mathrm{a}}$, Tatsushi

Nakamoto $^{\text {a }}$, Yasuhiro Makida ${ }^{\mathrm{b}}$, Masami Iio ${ }^{\mathrm{a}}$

${ }^{a}$ Applied Research Laboratory

KEK (High Energy Accelerator Research Organization)

1-1 Oho, Tsukuba, Ibaraki, 305-0801, Japan

${ }^{\mathrm{b}}$ Institute of Particle and Nuclear Studies

KEK (High Energy Accelerator Research Organization)

1-1 Oho, Tsukuba, Ibaraki, 305-0801, Japan

${ }^{c}$ Department of Applied Quantum Physics and Nuclear Engineering

Kyushu University

744 Motooka, Nishi-Ku, Fukuoka 819-0395, Japan 


\begin{abstract}
At J-PARC (Japan Proton Accelerator Research Complex), the COMET (Coherent Muon to Electron Transition) experiment is being prepared. Pion-capture and muontransport superconducting solenoid magnets will be used to capture pions and transport muons which are produced by pion decays. Since the radiation level is high near the magnets, a cold box and a current lead box, which supply a two-phase forced helium flow and currents to the magnets, will be located apart from the magnets. To connect the cold box and current lead box to the magnets, unique cryogenic transfer tubes that have thermal joints for installing $\mathrm{NbTi}$ superconducting lead wires at the insides of the transfer tubes are necessary. The transfer tubes should be able to make the wires thermally stable. In this paper, thermal contact conductance between three round-shaped $\mathrm{NbTi} / \mathrm{Cu}$ monolith superconducting wires and a rectangular slot in a copper block is measured from $4.5 \mathrm{~K}$ to $10 \mathrm{~K}$ in six cases and a concept of a thermal joint is proposed for the unique transfer tube of the muon-transport superconducting solenoid magnet. In the thermal joint, the round-shaped superconducting wires make line contacts with the slot covered with one layer of a Kapton tape. The void volume in the slot is filled with Apiezon $\mathrm{N}$ grease. An experimental apparatus that uses liquid helium is utilized for measuring the thermal contact conductance in a sample of the thermal joint. The effect of thermal cycles on the thermal contact conductance is observed. The measured thermal contact conductance is used to determine the length of the thermal joint and the size of the gap between the thermal joints.
\end{abstract}


Keywords: Round-shaped superconducting wire, thermal contact conductance, transfer tube, cryogenics, thermal joint

\section{Introduction}

At J-PARC, the COMET experiment is being prepared to observe the muon to electron conversion process which is one of charged lepton flavor violations. To control particles, superconducting magnets will be used at high radiation conditions. In a pion-capture superconducting solenoid magnet, a rectangular-shaped $\mathrm{NbTi}$ superconducting cable is used as a conductor to make a solenoid magnetic field, which captures pions produced by proton bombardments. In a muon-transport superconducting solenoid magnet, a round-shaped $\mathrm{NbTi}$ superconducting wire is used as a conductor and it transports muons generated by pion decays to a stopping target [1]. During 280 days operation (when cool-down and warm-up time is ignored), 0.8 MGy is estimated as a peak dose for the pion-capture magnet. Since the superconducting magnets are cooled down by using a conduction-cooling method with a two-phase forced helium flow, transfer tubes which connect a cold-box to the magnets are necessary. To supply currents to the superconducting magnets, a current lead box with superconducting current leads will be installed. Due to high radiation conditions near the magnets, the cold box and current 
lead box should be located apart from the magnets to avoid the effect of irradiation on the components of the cold box and current lead box. To supply simultaneously the twophase forced helium flow and currents to the magnets, unique cryogenic transfer tubes which have thermal joints for installing superconducting lead wires on $4.5 \mathrm{~K}$ return pipes are necessary [1]. Fig. 1 shows a schematic diagram that explains how to connect the magnets to the current lead box and the cold box.

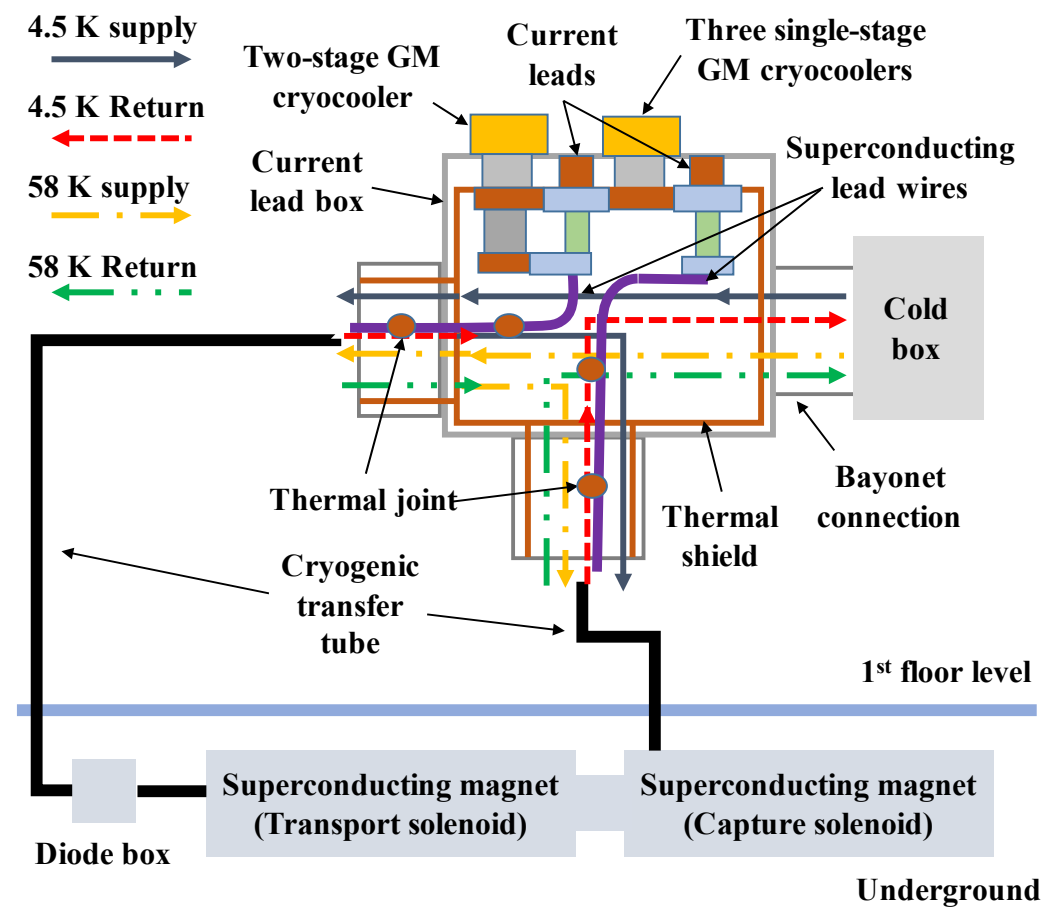

Fig. 1. Schematic diagram of connection between magnets and current lead box. 
For the thermal joints of superconducting lead wires, the most important factor is about thermal contact conductance between superconducting wires and a cold source. The thermal contact conductance is determined by joint methods and there are four kinds of joint methods, soldering, epoxy, grease, and dry joints [2]. In a conduction-cooling case, defective contacts between superconducting lead wires and a cold source generate the temperature increase, which results in the increase of cooling load or decrease of performance. The thermal contact problem happens more frequently in cryogenic systems. Most research is about the measurement of thermal contact conductance between plate-type materials. In our case, a round-shaped superconducting wire is used in the transfer tube for the transport magnet as current leads. It is preferred to use a thermal grease for the thermal joints due to fabrication process and maintenance.

In this paper, thermal contact conductance between three round-shaped $\mathrm{NbTi}$ superconducting wires and a rectangular slot in a copper block is measured to apply the thermal joint to the cryogenic transfer tube of the muon-transport superconducting magnet. The surfaces of the slot are covered with one layer of a Kapton tape (1205, 3M) and the void volume between the three round-shaped wires and the slot is filled with Apiezon $\mathrm{N}$ grease (M\&I Materials). In order to measure the thermal contact 
conductance between the three round-shaped superconducting wires and the slot from 4.5 $\mathrm{K}$ to $10 \mathrm{~K}$, an experimental apparatus that uses liquid helium and has very small heat leaks is utilized. At a sample joint, the thermal contact conductance is measured at six cases and the effects of Apiezon $\mathrm{N}$ grease and thermal cycles on the thermal contact conductance are observed. With the measured thermal contact conductance, the dimensions such as the length of the thermal joint and the size of the gap between the thermal joints are determined.

\section{Thermal joint for transfer tube of transport superconducting magnet}

2.1. Requirements of thermal joint with round-shaped superconducting lead wires

For the transport magnet, currents of $210 \mathrm{~A}$ and $165 \mathrm{~A}$ are required for main solenoid coils and dipoles, respectively, which means that four current leads are necessary. In the current lead box, four copper and superconducting current leads are cooled down by a two-stage GM-type cryocooler (SRDK-408D2, Sumitomo Heavy Industries, Ltd.) and cold ends of the four superconducting current leads are connected with superconducting lead wires. Thermal joints with the superconducting lead wires are installed on a $4.5 \mathrm{~K}$ return pipe of the transfer tube from the current lead box to the transport magnet. This is for avoiding the installation of an additional cooling system that preserves the 
temperature of long superconducting lead wires. The total length of the transfer tube is about $11 \mathrm{~m}$. The thermal joints installed on a $4.5 \mathrm{~K}$ return pipe should be thermally and electrically stable during the long operation along the transfer tube because it will be difficult to repair the thermal joints when the magnets operate and residual radioactivity exists. The configuration and mass of the thermal joint should be essentially considered to design the transfer tube because those factors determine the stress and deformation of a $4.5 \mathrm{~K}$ return pipe and configurations of spiders installed at the $4.5 \mathrm{~K}$ region.

Basically, we want to use a thermal grease in the thermal joints for the convenience of fabrication and modification and for allowing slips on surfaces during cooldown. If a semi-permanent soldering joint or epoxy joint is used, it will be difficult to modify the thermal joint when a problem is generated from the thermal joint. In that case, we need to cut the superconducting lead wires and it induces unnecessary joint resistances. For the thermal joints, we also want to use the round-shaped superconducting wire which is used for the solenoid coils of the transport superconducting magnet. Table 1 shows the specification of the superconducting wire. There are three reasons why the roundshaped superconducting wire is used. First, all of the vertical planes of straight sections in the transfer tube of the transport magnet are not in the same plane. It means that if a 
rectangular-shaped superconducting wire is used, we need to twist the rectangularshaped wire to maintain a direction of surface contact in a thermal joint. In the case of the round-shaped wire, we don't need to twist the round-shaped wire. Second, the roundshaped superconducting wire makes a line contact with a surface, so it will be easy to make that slip on a contacted surface when a $4.5 \mathrm{~K}$ return pipe and the superconducting wire are deformed at a cryogenic temperature. However, this induces a weak thermal contact condition. Therefore, it is required to exactly measure the thermal contact conductance of the joint in order to check the thermal stability of the joint. The last reason is because of the cost efficiency. We want to use the remaining superconducting wire after winding the round-shaped superconducting wire for the coils of the transport magnet.

\section{Table 1}

Specification of round-shaped superconducting wire.

\begin{tabular}{ll}
\hline Items & Values \\
\hline Conductor & $\mathrm{NbTi} / \mathrm{Cu}$ monolith wire \\
& $\mathrm{Cu}: \mathrm{NbTi}=6: 1$ \\
Cable dimensions & $\phi 1.57 \mathrm{~mm}$ (with insulation) \\
Cable insulation & Polyimide-imide enamel (AIW) \\
RRR & 150 \\
\hline
\end{tabular}

\subsection{Design of thermal joint}




\subsubsection{Concept of thermal joint}

A body material of the thermal joint is copper because the thermal expansion coefficient of copper is similar with that of stainless steel $304 \mathrm{~L}$ or $316 \mathrm{~L}$, which is the material of a $4.5 \mathrm{~K}$ return pipe in the transfer tube. There are two sections of bolted joints between a $4.5 \mathrm{~K}$ return pipe and the superconducting wires. One section of the bolted joints is between a $4.5 \mathrm{~K}$ return pipe and a body of the thermal joint. The other section is between the superconducting wires and the body of the thermal joint. The reasons why we use the two sections of bolted joints are 1. to control joint force for each joint section, 2. to make easy assembly; first, the body of the thermal joint is fixed on a $4.5 \mathrm{~K}$ return pipe and then superconducting wires are installed on the body. Even though a $4.5 \mathrm{~K}$ return pipe does not have an exact round shape, the first joint has relatively high thermal contact conductance [2] and the enough actual heat transfer area. Therefore, the thermal joint consists of three parts, a bottom body, a wire body, and a cover (first joint: bottom body and wire body, second joint: wire body with superconducting wires and cover). Since we need four current sections on a wire body of the thermal joint, there are four rectangular-shaped slots on the wire body. At each slot, three round-shaped superconducting wires will be installed for one current. Even though the superconducting wire has the insulation layer on itself, one layer of the Kapton tape is 
attached around the slot. Only one layer of the Kapton tape is enough to make the electrical isolation condition stable at the maximum voltage of the transport magnet. The void volume between the slot and superconducting wires is filled with Apiezon $\mathrm{N}$ grease. Therefore, the thermal paths made by the grease between the superconducting wires and surfaces of the slot are very important for the thermal joint. Also, it is necessary to check the stability of the thermal joint with Apiezon $\mathrm{N}$ grease during thermal cycles.

To determine the dimensions of the slot, we need to consider the total thickness of the Kapton tape and grease between the wires and surfaces of the slot. Fig. 2 shows the size and structure of the slot. The width and height of the slot are $5.2 \mathrm{~mm}$ and $1.8 \mathrm{~mm}$, respectively. We assume that the minimum thickness of the grease to y direction is 0.08 $\mathrm{mm}$ which is determined by the joint force, quantity of grease, and sizes of real gaps between the wires and surfaces of the cover and wire body. We assume that the minimum thickness of the grease of $\mathrm{x}$ direction is $0.09 \mathrm{~mm}$ (1. gaps between wires and 2 . gaps between wires and side surfaces) to easily install wires on the slot. At normal bolted joints, the joint force is an important factor in the thermal performance because the true contact area is increased in physical contact surfaces of two solids. However, 
we do not want to make high pressure on the superconducting wires and we just want to increase the areas of thermal paths by using the grease of the minimum thickness because we want to allow the superconducting wires to slip on the slot, easily and avoid making physical damage to the wires.

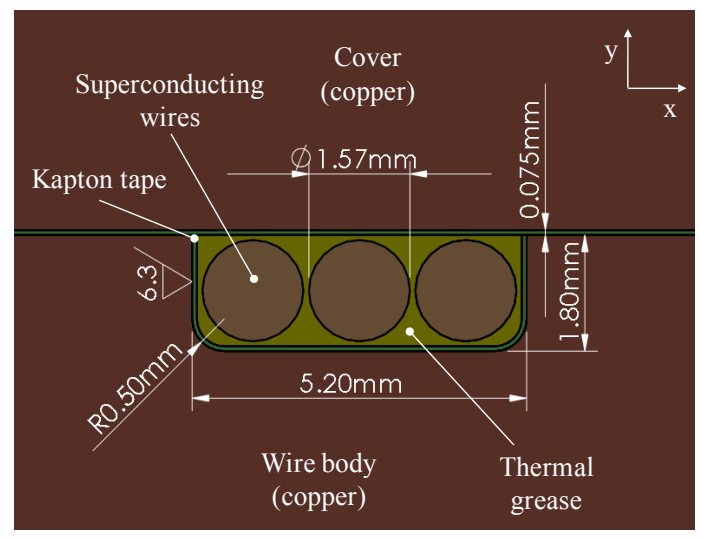

Fig. 2. Size and structure of slot for thermal joint of round-shaped superconducting wires.

\subsubsection{Sample of thermal joint for measurement}

We make a sample of the thermal joint to measure the thermal contact conductance at cryogenic temperatures. Fig. 3 shows a configuration of the sample joint. The sample joint consists of five parts (a copper block 1, a copper block 2, a copper block 3 with the rectangular slot, a copper cover, and the three round-shaped superconducting wires). The sample joint just has one slot for three superconducting wires. Since our thermal 
joint does not primarily depend on the joint force and temperatures of copper bodies are almost uniform, we can assume that one slot is thermally independent from the other slots. Therefore, we just need one slot on the sample to standardize the thermal contact conductance of the thermal joint which has round-shaped superconducting wires in a rectangular slot. Copper blocks 1 and 2 are soldered with the three superconducting wires. The copper block 2 is installed to measure temperature of the superconducting wires before the thermal joint. The minimum distance between the thermal joint and copper block 2 is $2 \mathrm{~mm}$ long. The temperature difference between copper blocks 2 and 3 is used to calculate the thermal contact conductance with Eq. (1).

$$
Q=h_{c} A_{c}\left(T_{2}-T_{3}\right)
$$

Where $Q$ is heat input on the copper block $1, h_{c}$ is the thermal contact conductance, $T_{2}$ is the temperature of the copper block $2, T_{3}$ is the temperature of the copper block 3 (it is the representative temperature of $h_{c}$ ), and $A_{c}$ is the total surface area of slot walls in the copper block 3 .

The temperature differences generated by the soldering and $2 \mathrm{~mm}$-long distance are 
negligible due to the high RRR value of the wires and the high thermal contact conductance of soldering. A film heater is installed on the copper block 1 . The total surface area on the slot is $4.75 \mathrm{~cm}^{2}$. To fill the void volume in the slot of the sample, the required mass of Apiezon $\mathrm{N}$ grease is ideally $0.09 \mathrm{~g}$. But, it is difficult to fill the void volume with the grease, completely. Also, the Kapton tape can make void volume at the edges of $0.5 \mathrm{~mm}$ fillets because it is difficult to completely attach the Kapton tape on the edges. The real length of the thermal joint and the gap size between the thermal joints are determined by the measured thermal contact conductance $\left(h_{c}\right)$ between the superconducting wires and the slot of the sample joint.

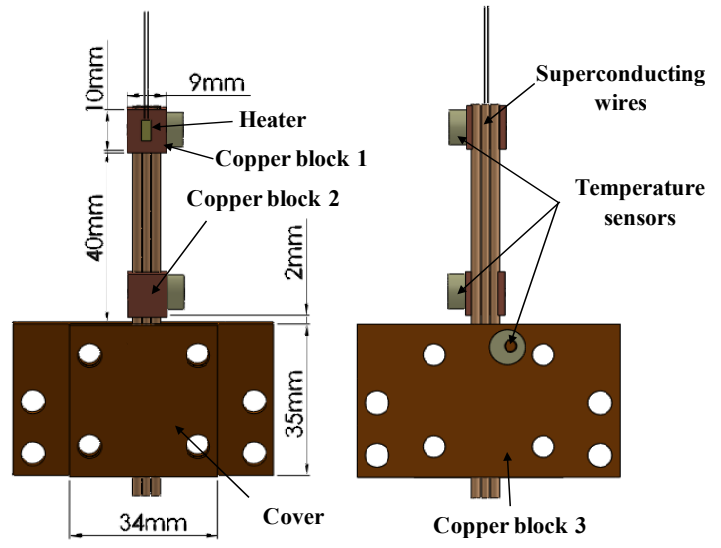

(a) Front and back views

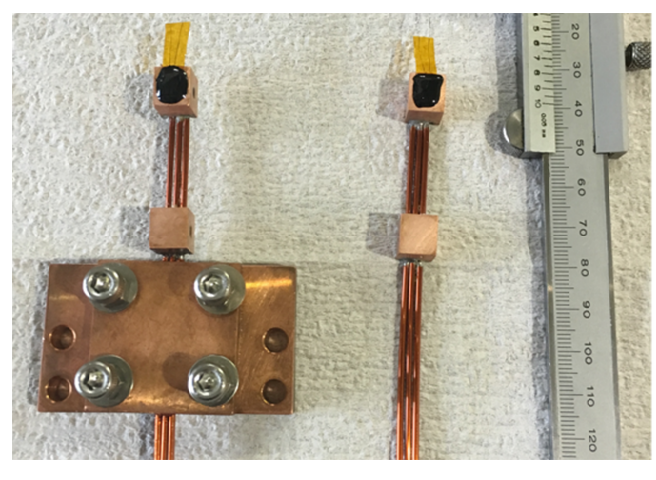

(b) Photo

Fig. 3. Configuration of sample joint. 


\subsection{Pressure distribution}

Since a real thermal joint has four slots and it is difficult to make contact pressure high at the middle two slots, the sample joint should have weak pressure contacts between the superconducting wires and surfaces of the slot. In order to check the contact pressure between wires and the surface of the cover in the sample joint, we used a thin pressure films (Prescale, Fujifilm). We used $1.5 \mathrm{~N} \cdot \mathrm{m}$ torques for joints of M5 bolts on the sample joint. In the future, the results of pressure distribution will be referred to make physical contact conditions of slots in a real thermal joint similar to that of the slot in the sample.

Fig. 4 shows pressure distribution in the sample joint. We changed the quantity of Apiezon $\mathrm{N}$ grease in the slot of the sample and checked the contact pressure by using the film. Since the superconducting wire is not completely straight, there are partial contacts between the wires and the surface. If the quantity of grease increases, the amplitude of pressure or the distributed area of pressure increases. This is normal, but this also depends on distribution of the grease on the slot. When we insert the grease in the slot, it is difficult to make the grease uniformly distributed along the slot and to make the grease same in every slot. Therefore, it can make the error of reproducibility for the measurement of thermal contact conductance. From the test of pressure distribution, we confirmed that super low pressure (LLW: $0.5-2.5 \mathrm{MPa}$ ) was applied to 
the wires in the cases of $\mathrm{b}$ and $\mathrm{c}$. When the void volume was not filled with the thermal grease (case b), the superconducting wires had partially line contacts with the slot. In case c (filled with $0.09 \mathrm{~g}$ grease), the level of contact pressure was similar to that of case $b$, but the contact area was increased due to the effect of the thermal grease. When we overcharged the void volume with the thermal grease (case d), the contact pressure condition was the level of low pressure (LW: $2.5 \mathrm{MPa}-10 \mathrm{MPa}$ ) on the line contacts. The joint pressure is important for normal thermal joints. But, for our joint, we do not want to apply high pressure to the superconducting wires and we just want to widen contact areas by using the thermal grease. $0.09 \mathrm{~g}$ is determined as a standard mass of Apiezon $\mathrm{N}$ grease in the slot of the sample joint.

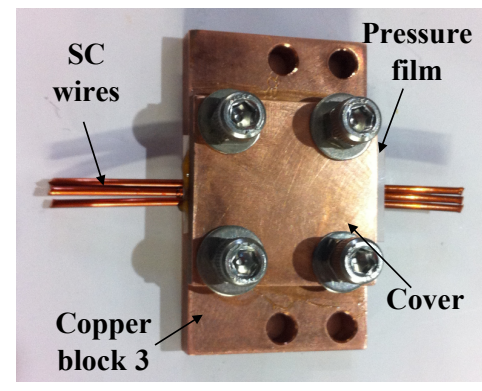

(a) Joint for checking pressure distribution

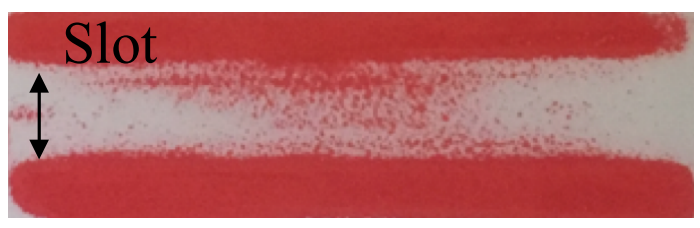

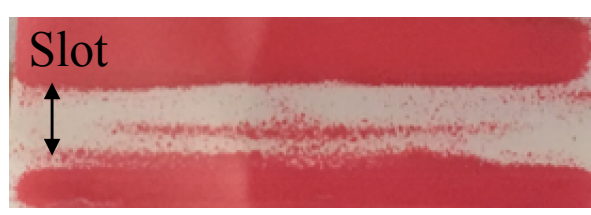

(b) Without grease (LLW film)

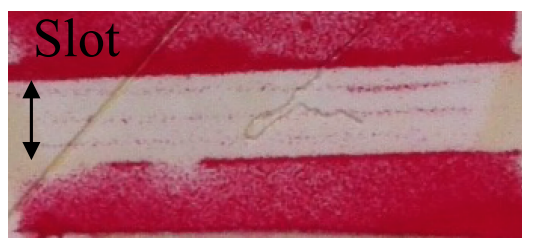


(c) With $0.09 \mathrm{~g}$ grease (LLW film)

(d) With 0.2 g grease (LW film)

Fig. 4. Pressure distribution of sample joint

(On the film, spots mean the area is pressurized).

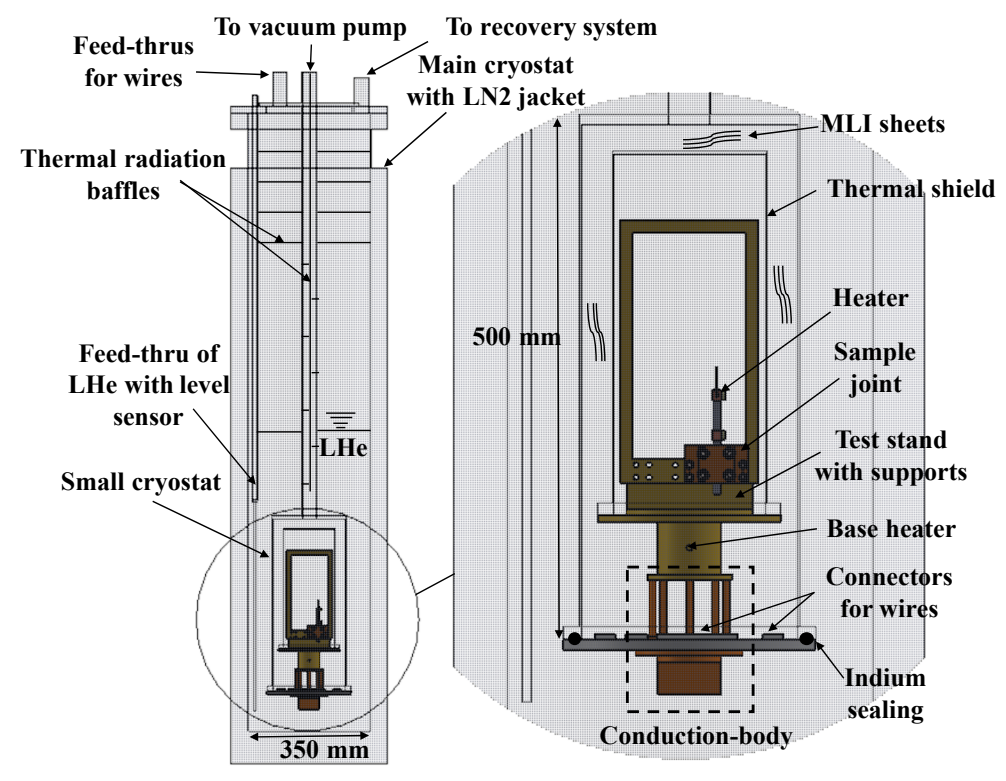

Fig. 5. Experimental apparatus for measuring thermal contact conductance.

\section{Experiments}

\subsection{Experimental apparatus}

Fig. 5 shows an experimental apparatus to measure the thermal contact conductance of the sample joint from $4.5 \mathrm{~K}$ to $10 \mathrm{~K}$. The sample joint is installed on a test stand and the 
copper block 3 of the sample joint is conductively cooled down by a conduction-body.

The conduction-body contacts with liquid helium and the temperature of the test stand is controlled by a base heater. The test stand has supports to thermally anchor electrical wires of temperature sensors and heaters. The test stand is surrounded by a thermal shield with MLI sheets and the thermal shield is also cooled by the conduction-body. The test stand is covered and sealed by a small cryostat which is at a high vacuum condition. The small cryostat is contained in a main cryostat. The main cryostat has a LHe level sensor to measure the level of liquid helium and the maximum level of liquid helium is 47 inch high. Vaporized helium gas is recovered to a recovery system.

\subsection{Measurement}

Calibrated Cernox sensors (CX-1050-CU, Lake Shore) were used to measure all temperatures. Temperatures of copper blocks 2 and 3 were used to calculate the thermal contact conductance between superconducting wires and the rectangular slot. All temperatures were monitored by a temperature monitor (Model 218, Lake Shore) and transferred to a DAQ system. A stain gauge (KFG-1-120-C1-11, KYOWA) was used for a heater which is installed on the copper block 1. Fig. 6 shows a thermal circuit of conductive heat transfer for the sample joint. The total heat leak $\left(0.68 \%\right.$ of $Q_{i n}$ from 
wires of heater and $0.02 \%$ of $Q_{i n}$ from wires of temperature sensors) from thermal conduction is very small because the diameters (32 AWG for the heater and 36 AWG for temperature sensors) and thermal conductivities of the electrical wires are much smaller than those of the superconducting wires. The heat leak from thermal radiation is ignored because the temperature differences between the sample and others are small (below than $7 \mathrm{~K}$ ) and the thermal shield and test stand are coated with Au. Once all of the temperatures were stable and the level of liquid helium was reached to the maximum level (47 inch), we started our measurement. Normally, we just inputted heat to the heater installed on the copper block 1. It increased all temperatures of the sample and made the temperature difference between the superconducting wires and the slot. When we wanted to reduce the temperature difference, first, temperature of the copper block 3 is controlled by the base heater installed on the test stand, and then heat (smaller than heat at the normal way) is inputted to the copper block 1. 


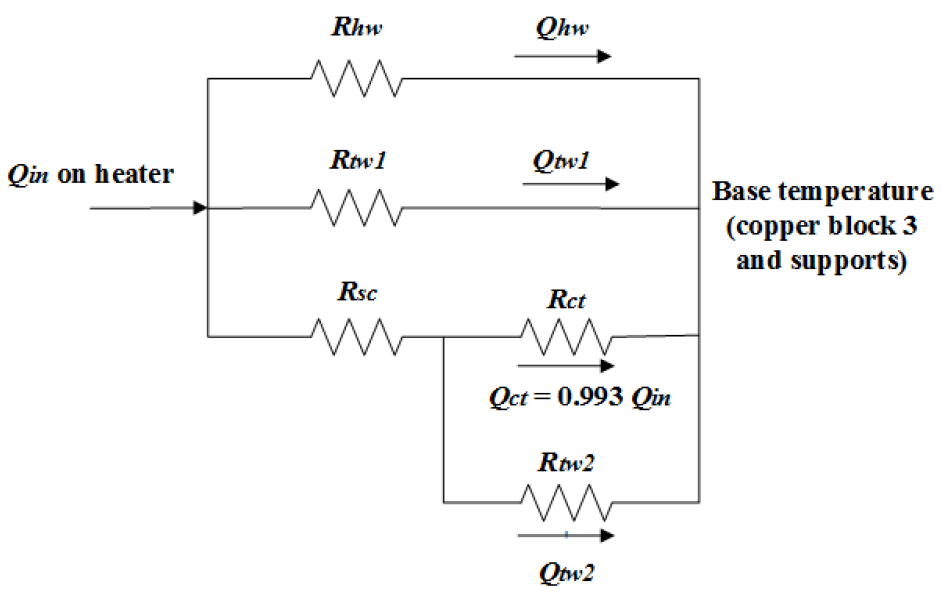

Fig. 6. Thermal circuit of conductive heat transfer ( $R$ : thermal resistance, $h w$ : wires of heater, $t w$ : wires of temperature sensors, $s c$ : superconducting wires, $c t$ : thermal contact - we assume $R_{c t}=1 / 0.005(\mathrm{~K} / \mathrm{W})$, and $Q$ : heat $)$.

\subsection{Cases}

We made six samples of superconducting wires. Each sample was installed on the slot of the copper block 3 and tested in the experimental apparatus. There are six cases and Table 2 shows the conditions of cases. The thermal contact conductance is dependent on the condition of the thermal grease. When the thermal grease is uniformly filled in the void volume of the slot with the minimum thickness of the thermal grease, it will make the best thermal performance. If the thermal grease is overcharged in the slot, the distances between superconducting wires and surfaces of the slot are increased and it induces unnecessary thermal resistance (the thermal conductivity of Apiezon $\mathrm{N}$ grease is 
not good at cryogenic temperatures [3]). However, it is difficult to evenly distribute the thermal grease in the slot and make every joint in the same condition. The reason why we changed the mass of the thermal grease is to check the effect of the quantity changes of the thermal grease on the thermal contact conductance and to confirm the error of reproducibility. Apiezon $\mathrm{N}$ grease withstands thermal cycling between cryogenic and room temperatures [4]. At two cases (case 5 and case 6), 21 thermal cycles were applied to each case to check the stability of Apiezon $\mathrm{N}$ grease during the thermal cycles. The sample joint with superconducting wires was inserted in a bath of liquid nitrogen. When the temperature of sample was stabilized, the sample joint was pulled out from the bath and heated up to $42{ }^{\circ} \mathrm{C}$. The thermal cycles can eliminate the overcharged grease from the slot. While the slot of the sample joint shrinks, if the shrinkage of the slot (copper) is larger than the total shrinkage of Kapton tapes and thermal grease, it pulls out the overcharged thermal grease from the slot. When the temperature of the thermal grease reaches near the dropping temperature $\left(42{ }^{\circ} \mathrm{C}-52{ }^{\circ} \mathrm{C}\right)$ of the grease, the overcharged thermal grease easily flows out from the slot. The effect of the thermal cycles decreases the mass of thermal grease in case 5 . Thermal cycles between $300 \mathrm{~K}$ and $77 \mathrm{~K}$ can possibly create cracks in the grease or separation of the grease [3]. If Apiezon $\mathrm{N}$ grease traps gas at the inside of the grease and is contaminated by gas during thermal cycles, it 
makes cracks in the solidified grease or the separation of the grease. We randomly inserted the thermal grease in the slot at the cases 4 and 6 .

\section{Table 2}

Cases in measurement.

\begin{tabular}{lll}
\hline Cases & Mass of Apiezon N grease $(\mathrm{g})$ & Thermal cycles \\
\hline 1 & 0 & 0 \\
2 & 0.09 & 0 \\
3 & 0.11 & 0 \\
4 & Random & 0 \\
5 & 0.09 (after thermal cycles: 0.07$)$ & 21 \\
6 & Random & 21 \\
\hline
\end{tabular}

\section{Experimental results}

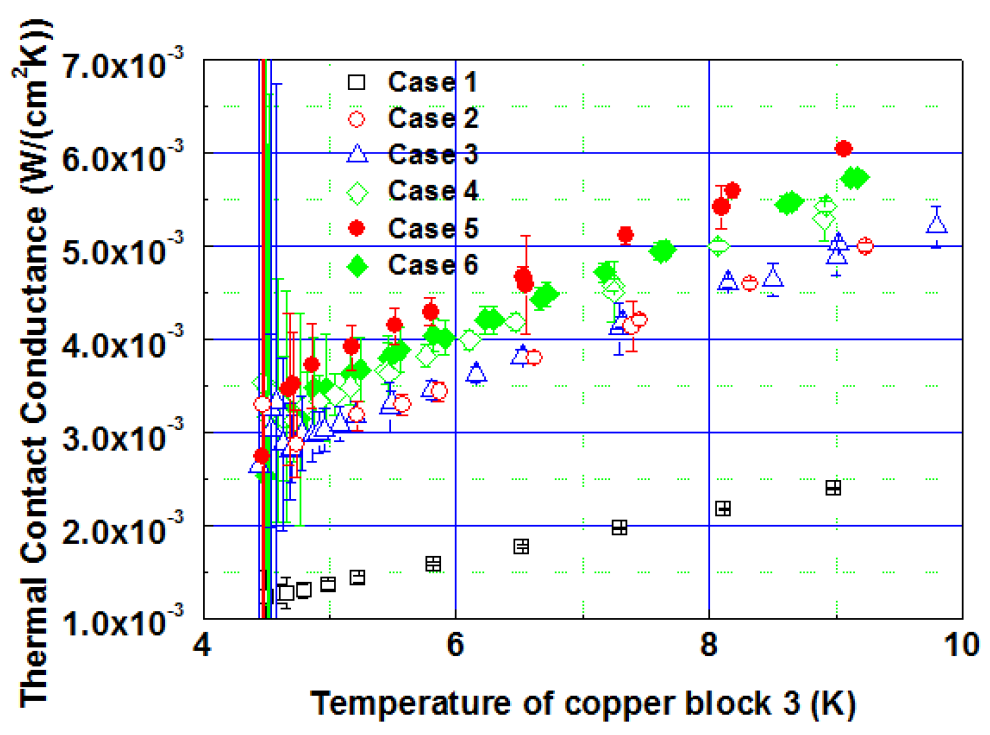

Fig. 7. Thermal contact conductance of thermal joint as a function of temperature. 
In order to normalize the thermal contact conductance, overall thermal contact conductance (unit: $\mathrm{W} / \mathrm{K}$ ) of the sample joint is divide by the total area $\left(4.75 \mathrm{~cm}^{2}\right)$ of the slot surfaces. Fig. 7 shows the normalized thermal contact conductance calculated from the measured temperature differences. It is confirmed that the cases $2-6$, which are filled with Apiezon $\mathrm{N}$ grease, have higher thermal contact conductance than that of case 1. In our measurement, the major errors are generated from the accuracies of calibrated Cernox sensors. The typical accuracy of Cernox sensor is $\pm 5 \mathrm{mK}$ at $4.2 \mathrm{~K}$. If the temperature differences between the copper blocks 2 and 3 are small, the effects of the accuracies of Cernox sensors on the thermal contact conductance are increased and those make large error bars. Our experimental system does not have a large cooling capacity at around $4.5 \mathrm{~K}$. That is the reason why the error bars are very large at around 4.5 $\mathrm{K}$ of the cases $2-6$. However, without the points at around $4.5 \mathrm{~K}$, the tendencies of points of thermal contact conductance are linear $\left(h_{c} \approx a T, a\right.$ : coefficient of proportionality, $T$ : temperature), so the issue does not make a problem. At some points between $5 \mathrm{~K}$ and $10 \mathrm{~K}$, error bars are relatively larger than those at other points. It is because of the heat input to the base heater. The temperature of the copper block 3 is the representative temperature of thermal contact conductance. At normal measurement, we 
inputted heat to the heater installed on the copper block 1. It made relatively large temperature differences between the copper blocks 2 and 3 from $5 \mathrm{~K}$ to $10 \mathrm{~K}$. To check the representativeness of temperature of the copper block 3 , we inputted heat to the base heater and decreased the temperature differences at some points (as controlled points by using the base heater) of cases 2-5. It is confirmed that the thermal contact conductance at some points (controlled points) is similar to that at normal points.

Another issue is about the reproducibility of the thermal joint. We cannot make every thermal joint identical. It is estimated that the reproducibility of the sample joint is around $10 \%$ when the results from case 2 to case 4 are compared. There is an interesting result about the effect of the thermal cycles on the thermal contact conductance. Cases 5 and 6 have the higher thermal contact conductance than other cases because the thermal cycles make the distribution of Apiezon $\mathrm{N}$ grease uniform and decrease the thicknesses of the thermal grease between the superconducting wires and surfaces of the slot and cover. Therefore, it is confirmed that the effective thickness and distribution of the thermal grease are important for application of the thermal grease at cryogenic temperatures and the thermal cycles can help to increase the thermal contact conductance for the thermal joint with the thermal grease. Also, the effects of cracks in 
the solidified grease and separation of grease are not observed in the sample joint to which 21 thermal cycles are applied.

\section{Simulation for prediction of temperature of SC wires}

To determine the length and the gap size of a real thermal joint for the transfer tube, the worst number (obtained at the case 1) of thermal contact conductance is used in ANSYS. Table 3 shows the setting values in the steady-state thermal analysis of ANSYS. In this simulation, we assume that the volume of four slots is completely filled with the material of superconducting wires and the temperature differences between the superconducting wires and surfaces of slots are generated by the worst number of thermal contact conductance and heat input. Fig. 8 shows the configuration of a real thermal joint and the estimated temperature condition of the real thermal joint in ANSYS. The length of the thermal joint is $50 \mathrm{~mm}$ long and the gap size between the thermal joints is $200 \mathrm{~mm}$ long. Since the highest temperature is near $4.8 \mathrm{~K}$ (when the thermal contact conductance between the copper body and cover is $100 \mathrm{~W} /\left(\mathrm{m}^{2} \cdot \mathrm{K}\right)$, the highest temperature is $4.75 \mathrm{~K}$ ) in the thermal analysis, the length and gap size will make the superconducting wires thermally stable. If the four slots in the real thermal joint can have the similar contact pressure with the slot of the sample joint, it will be enough to 
make a suitable heat transfer condition for the real thermal joint. In the future, the locations and sizes of bolts will be tuned by using the test of pressure distribution.

\section{Table 3}

Setting values in ANSYS.

\begin{tabular}{ll}
\hline Cases & Values \\
\hline Material of SC wires & RRR $=150$ (copper) \\
Copper body with slots and copper cover & RRR $=30$ \\
Radiative heat flux & $0.25 \mathrm{~W} / \mathrm{m}^{2}$ \\
Heat generation due to irradiation & $0.01 \mathrm{~W} / \mathrm{kg}^{\mathrm{a}}$ \\
Temperature of heat sink & $4.6 \mathrm{~K}$ \\
Thermal contact conductance on surfaces of slot & $10 \mathrm{~W} /\left(\mathrm{m}^{2} \cdot \mathrm{K}\right)^{\mathrm{b}}$ \\
Thermal contact conductance between copper body and cover & $10-100 \mathrm{~W} /\left(\mathrm{m}^{2} \cdot \mathrm{K}\right)^{\mathrm{c}}$ \\
\hline
\end{tabular}

${ }^{\mathrm{a}}$ It is equal to $0.01 \mathrm{~Gy} / \mathrm{sec}$ and corresponds with a radiation dose of $0.24 \mathrm{MGy}$ for the experiment life time. In the transfer tube, the estimated dose is much less than $0.1 \mathrm{MGy}$.

${ }^{b}$ To consider unexpected errors and contingency generated in fabrication, the worst number (case 1) of thermal conductance is used.

${ }^{c}$ The highest value is obtained from $h_{c}=$ thermal conductivity of Kapton tape $(0.01 \mathrm{~W} /(\mathrm{m} \cdot \mathrm{K})$ at $4.5 \mathrm{~K}) /$ thickness of Kapton tape $(0.000075 \mathrm{~m})$ with a 1.33 safety factor and the lowest value is obtained from the worst number of the measured thermal conductance.

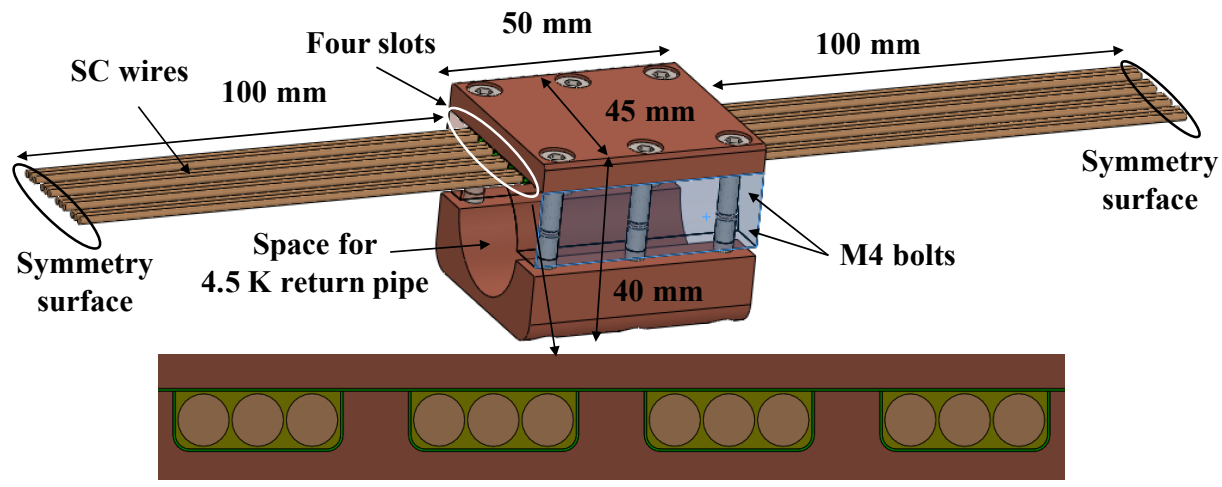

(a) Configuration of real thermal joint (total mass of joint : $\sim 0.6 \mathrm{~kg}$ ) 

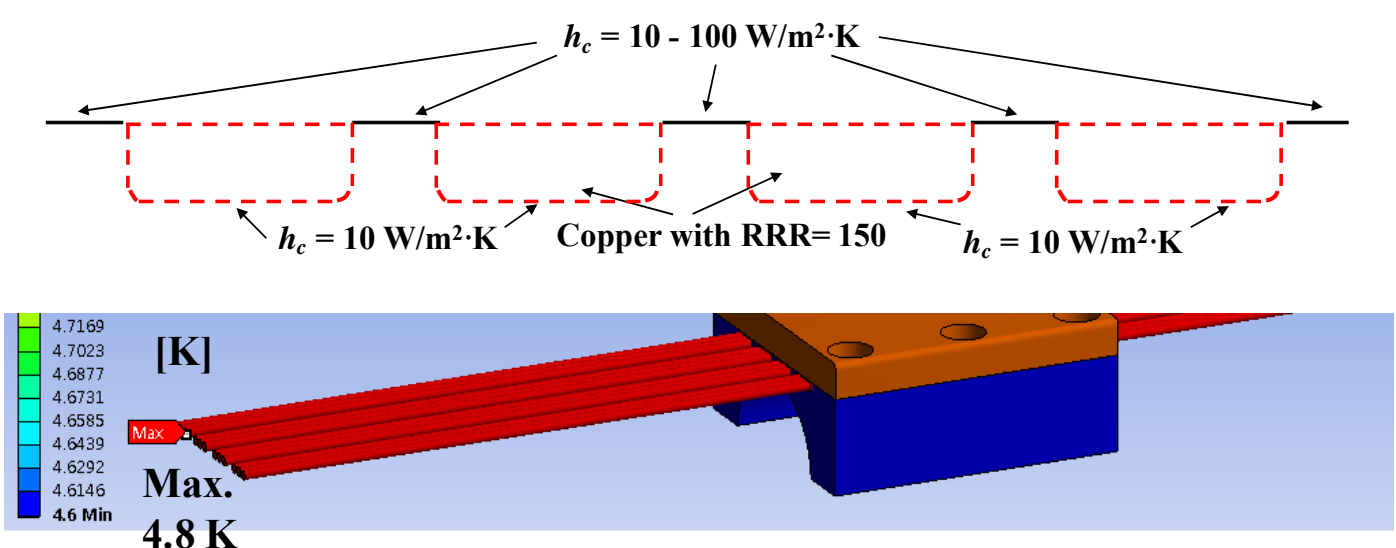

(b) Configuration of thermal joint and estimated temperature condition in ANSYS

(thermal contact conductance between copper body and cover $=10 \mathrm{~W} /\left(\mathrm{m}^{2} \cdot \mathrm{K}\right)$ )

Fig. 8. Configuration of thermal joint and temperature condition.

\section{Discussion}

In the COMET experiment, neutrons penetrate particle-radiation shields surrounding a target and affect materials of magnets and cryogenic systems. Apiezon $\mathrm{N}$ grease is a kind of hydrocarbon grease without silicones. Since the neutron is reacted with hydrocarbons, neutron may influence properties of Apiezon $\mathrm{N}$ grease and irradiation can generate heat in the thermal joint. At the transfer tube with the thermal joints, a radiation dose for the experiment life time is much less than $0.1 \mathrm{MGy}$, which is estimated by PHITS [5]. It is estimated that the radiation dose is very small and it cannot make a big 
effect on the thermal joint. But we need to check the effect of irradiation on Apiezon $\mathrm{N}$ grease, especially the effect on thermal performance of the grease, because the thermal grease is frequently used at cryogenic systems and someone may want to use it at a neutrons scattering experiment with cryogenic systems. Therefore, it is necessary to check the effect of neutrons on the thermal performance of Apiezon $\mathrm{N}$ grease with diverse radiation levels.

Since the measured thermal conductance is dependent on 1) thickness of the Kapton tape, 2) dimensions of wires, and 3) condition of thermal grease, the effect of them on the thermal conductance needs to be checked in detail. To make a map for the thermal conductance and to consider all of the dominant factors in Eq. (1), it is required to do more tests with other samples. As showed in Fig. 7, our result shows that the thermal cycles between $42{ }^{\circ} \mathrm{C}$ and the temperature of liquid nitrogen improve the thermal conductance. Since the thermal cycles make the distribution of the grease uniform and decrease the thickness of the grease, the thermal conductance is improved. However, it is difficult to say that heating to $42{ }^{\circ} \mathrm{C}$ heals the cracks and separation of the grease. To know the effect of the heating to $42{ }^{\circ} \mathrm{C}$ on the cracks and separation, it is necessary to do an experiment with a special sample which can visualize the condition of the grease. 
The performance test of the real thermal joint will be performed when the cryogenic transfer tube is fabricated. The test result will be included in a paper which explains about the development of unique transfer tubes for the COMET experiment.

\section{Summary}

In this paper, thermal contact conductance between three round-shaped superconducting wires and a rectangular slot in a copper block is measured at cryogenic temperatures and a thermal joint with four slots, which have three round-shaped superconducting wires at each slot, is designed for application to a cryogenic transfer tube. In the cryogenic transfer tube, the thermal joints are installed on a $4.5 \mathrm{~K}$ return pipe of the transfer tube along the length of $11 \mathrm{~m}$. The thermal joints should make the twelve superconducting wires thermally stable. In each slot of the thermal joints, three round-shaped superconducting wires are installed on each slot and are covered by a copper block. The surfaces of each slot are covered with one layer of a Kapton tape for electrical insulation and void volume between the round-shaped wires and the slot is filled with Apiezon $\mathrm{N}$ grease. By using an experimental apparatus with a sample of the thermal joint, the thermal contact conductance is measured from $4.5 \mathrm{~K}$ to $10 \mathrm{~K}$ in six cases. It is 
confirmed that Apiezon $\mathrm{N}$ grease effectively increases the thermal contact conductance between three round-shaped superconducting wires and the rectangular slot and thermal cycles also help to increase the thermal contact conductance. With the worst number (1 $\mathrm{x} 10^{-3} \mathrm{~W} / \mathrm{cm}^{2} \cdot \mathrm{K}$ ) of thermal contact conductance, a configuration of standard thermal joint is designed and working temperature is estimated in ANSYS.

\section{Acknowledgments}

This work was supported by Japan Science and Technology Agency under Strategic Promotion of Innovative Research and Development Program (S-Innovation Program) and Japan Society for the Promotion of Science Grants-in-Aid for Scientific Research (KAKENHI). And T. Ki was supported by Basic Science Research Program through the National Research Foundation of Korea (NRF) funded by the Ministry of Education,

Science, and Technology (2015R1A6A3A03018889).

\section{References}

[1] T. Ki, M. Yoshida, Y. Yang, et al., Cryogenic system for COMET experiment at JPARC, Cryogenics 77 (2016) 25-35. 
[2] S. W. Van Sciver, Helium cryogenics, second ed., Springer, New York, 2012, chapter 2 .

[3] M. M. Kreitman, J. T. Callahan, Thermal conductivity of Apiezon N grease at liquid helium temperatures, Cryogenics 10 (2) (1970) 155-159.

[4] Apiezon $\mathrm{N}$ cryogenic high vacuum grease technical data sheet, http://www. apiezon.com.

[5] T. Sato, K. Niita, N. Matsuda, et al., Particle and heavy ion transport code system PHITS - version 2.52, J. Nucl. Sci. Technol. 50 (9) (2013) 913-923. 